\title{
Micro-Force Sensor by Active Control of a Comb-Drive
}

\author{
Abdenbi Mohand Ousaid, Sinan Haliyo, Stéphane Régnier and Vincent Hayward
}

\begin{abstract}
This article describes an active force sensor with an accuracy of $0.4 \mu \mathrm{N}$ over a large range [-400 to 400$] \mu \mathrm{N}$. The mechanical structure, through a fiber suspension arrangement, provides exactly one degree of freedom. This design allows for a precise displacement sensing by a laser optical lever. The force sensing principle is based on the active control of an electrostatic bi-polar actuator. A meso-scale prototype has been built as proof-of-concept and an especially designed controller allows for high precision measurements on a large range. The system is validated by measuring both pull-in and pull-off forces during an approach-retract cycle on water droplet. Both phenomena, although differing by an order of magnitude in amplitude, are correctly assessed.
\end{abstract}

\section{INTRODUCTION}

Interaction forces play a key role in micro-scale probing, characterization, manipulation, and assembly systems. Their range, i.e dependence on the distance between interacting bodies, and magnitude vary greatly as a function of the intended application. For example in biology, the contractile forces in the $\mathrm{mN}$ range from isolated ventricular heart cells (cardiac myocytes) differs in normal or diseased heart [1]. In nanotechnologies, measurement of adhesion forces is required for experimental validation of different models for micro objects interactions, like model of adhesion developed in [2]. In microscale manipulation, those interaction forces affect greatly gripping and assembly strategies [3], [4].

Several types of micro and nano force sensors were developed to provide small force measurements. Most are based on a passive and indirect measurement, i.e through the deformation or displacement of a sensitive part with a calibrated stiffness. Most designs are based on elastic microstructures such as microcantilevers [5], piezoresistive sensors using the variation of resistance on a piezoelectric layerb [6], capacitive sensors based on the variation between two armatures over distance [7], direct piezoelectric sensors through the correlation between their deformation and produced current [8]

Nevertheless, the most widespread approach is the Atomic Force Microscopy (AFM) [9][10]. The deflection of a cantilever of known characteristics is measured by a two or four quadrants photodectectors [11] or by interferometry [12]). The applied force is then deduced using the measured deflection and the stiffness of the cantilever.

Despite their popularity, passive force measurements with cantilever-based probes have serious limitations. First, cantilevers are hard to calibrate. Geometry-dependent

The authors are with Institut des systèmes Intelligents et de Robotique, Université Pierre et Marie Curie, CNRS UMR 7222, 4 Place Jussieu, 75252 Paris Cedex, France \{mohand ousaid, haliyo, regnier, hayward\}eisir.upmc.fr probe/sample interactions cause the occurrence of a combination of bending and torsion modes that makes data interpretation difficult. AFMs, because of the Bernoulli assumption, also suffer from a restricted dynamic range when high accuracy is required. Other limitations comes from several non-linear phenomena, such as hysteresis and input saturation on optical levers. Perhaps the greatest limitation of the cantilevered beam is the unavoidable trade-off that must be made between stiffness, sensitivity, and structural response characteristics.

The concept of active force measurement principle or force balancing is introduced to overcome these limitation of passive systems, such as used in some accelerometers [13] and pressure sensors [14]. In those devices, an actuator is integrated to keep the moving part of the sensor in equilibrium position. In direct force sensing, an active sensors where the applied force is balanced using an electrostatic micro actuator is recently proposed [15]. Another sensor, based on cantilevered composite beam structure with embedded piezoelectric polyvinylidene fluoride (PVDF) actuating and sensing layers is mentioned [16]. However, this design still carries the trade-off related to the cantilevered beam stiffness, mentioned in the previous paragraph. To deal with this limitation, the application of the active force measurement principle to the sensing approach seems a promising solution [17] .

An improvement of the design of the device proposed in [17], along an adapted control scheme, is proposed in this paper. The systems uses electrostatic energy, which can be modulated at electronic speeds, instead of strain energy to act against external interaction forces. The probe can be represented by a lumped parameter system, that is, by rigid body motion, to dissociate the sensor stiffness from the force measurement. The movements of the probes are constrained along one single dimension by a fiber suspension that provides nearly perfect kinematic guidance with very low stiffness and virtually no friction. Precise guidance makes it possible to measure the displacement of the moving probe carrier by an optical lever. The suspended probe carrier includes a differential electrostatic actuator, a comb-drive. This actuator is used to balance the external force through the feedback loop and hence provides direct force measurement over the entire operating range.

\section{SYSTEM DESCRIPTION}

The proposed force sensor is designed around a moving probe kinematically constrained to a single translation motion, through a glass fiber suspension design, and carrying the moving part of a comb-drive armature. 


\section{A. Fiber suspension}

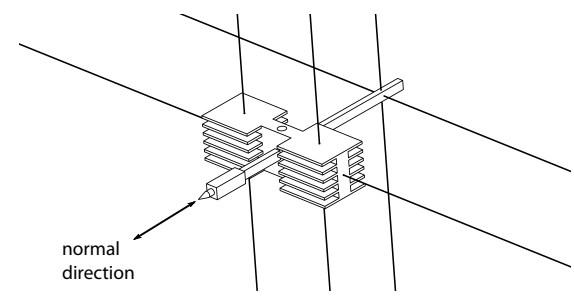

Fig. 1. Five-fiber suspension.

In order to constrain the motion of the probe to a single axis, a suspension with 5 glass fibers is designed, as depicted in Fig. 1. The length of fibers is much higher then their expected deformation. They are held at both extremities with a rocker-and-leaf mechanism allowing to manually adjust their tensions. It is safe to consider that the only possible motion for an object fixed in their middle is in a direction perpendicular to their axis, given that their axial stiffness is much higher then the bending one. Moreover, given the very low deformation-to-length ratio, this bending stiffness can be considered constant.

The 3 vertical fibers effectively constrain the motion of the carrier to planar motions. The 2 horizontal fibers add additional constrains and effectively limit the motion to a single translation. The suspension compliance is tuned by adjusting the tension of the fibers and the tension in the front fibers equalize spurious torque.

An optical lever, consisting of an external laser source, a mirror embedded on the carrier, and an external four quadrant photodiode is used to measure the displacement of the probe. The resulting system is shown in Fig. 2, including fiber fixations and the optical lever.

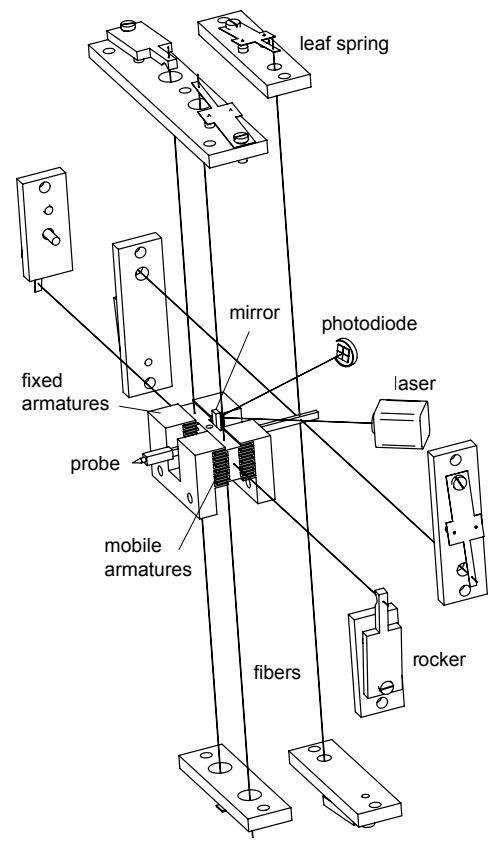

Fig. 2. Mechanical system.

\section{B. Differential bipolar comb actuator}

(2)

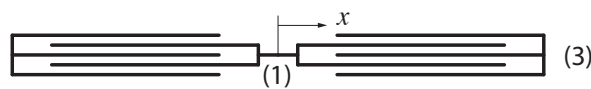

Fig. 3. Differential bipolar comb actuator.

A bipolar differential electrostatic actuator, called a combdrive, is used in the system to balance the external force. Fig 3 is a schematic representation of such an actuator.

A moving armature (1) is included in the carrier, and two fixed armatures (2) and (3) are mounted on the external frame. Given three voltages $v_{1}, v_{2}, v_{3}$ corresponding to the armatures (1), (2), and (3), the electrostatic energy is

$E=\frac{1}{2}\left(c_{11} v_{1}^{2}+c_{22} v_{2}^{2}+c_{33} v_{3}^{2}+2 c_{12} v_{1} v_{2}+2 c_{13} v_{1} v_{3}+2 c_{23} v_{2} v_{3}\right)$

where the $c_{i j}$ represent the capacities of armature pairs.

Considering $x$ the displacement of the moving armature, the actuator force is $\partial E / \partial x=\frac{1}{2} c_{n}\left(-v_{2}^{2}+v_{3}^{2}-2 v_{1} v_{2}+2 v_{1} v_{3}\right)$, where $c_{n}$ depends on the size of armatures and their gap, all considered equal. Controlling $v_{2}, v_{3}$ to be opposite in sign and equal in magnitude, assigning them to a control voltage $v_{n}$ such that $v_{3}=v_{n}, v_{2}=-v_{n}$, and fixing $v_{1}$, the force developed by the actuator is

$$
a_{n}=2 c_{n} v_{1} v_{n}
$$

Thus, the force provided by the actuator is proportional to $v_{n}$ and $v_{1}$ can be used to adjust the actuator constant.

It is important to note that this force does not depend on the position of the probe on the normal direction, as long as the overlapping surface on mobile and fixed armatures is constant. It is also linear in respect to control voltage.

This is a considerable benefit over flexible systems with intrinsic dependencies between force and displacement.

\section{Mechanical behavior}

The dynamic of the system is then well described by a second order differential equation involving all forces applied to the probe,

$$
f=m \ddot{x}-a_{n}-k x-b \dot{x}
$$

where $f$ is the interaction force between probe and sample, $m \ddot{x}$ is the inertial term, $a_{n}$ is the force provided by the actuator, $k x$ is the force due to suspension compliance, $b \dot{x}$ is the viscous damping term.

\section{Prototype}

The meso-scale prototype illustrated in Fig 4 is comprised a frame made of polyoxymethylene plastic (180 mm $\times 180 \mathrm{~mm} \times 40 \mathrm{~mm}, 10 \mathrm{~mm}$ thickness) supporting the fiber positioning and tensioning mechanisms and the fixed actuator armatures. The carrier is machined out of magnesium to minimize mass $(1.2 \mathrm{~g})$. Its dimensions are $22 \mathrm{~mm} \times 8.5 \mathrm{~mm} \times 7.4 \mathrm{~mm}$ and the gap between fixed and moving armatures of $0.3 \mathrm{~mm}$. The springs are made out pinchbeck. Care was exercised to minimize hysteresis by 

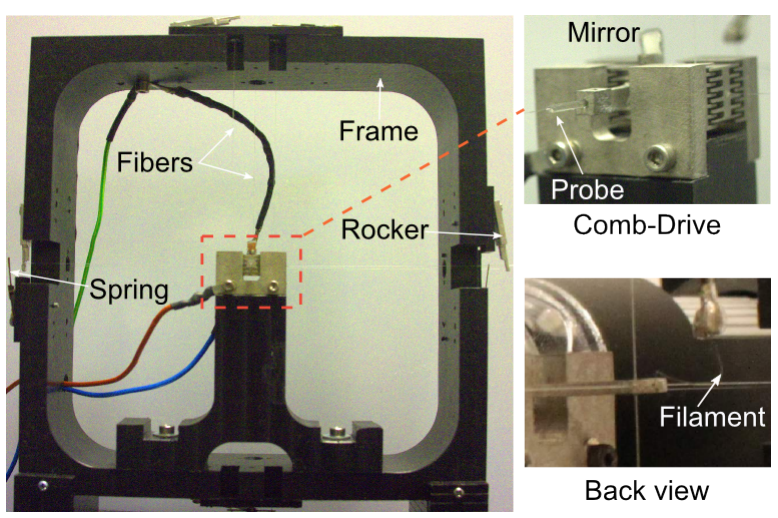

Back view

Fig. 4. Mechanical elements.

using long glass fibres (180 mm longer, $0.1 \mathrm{~mm}$ diameter) and careful fiber attachment design. The probe is made from pulled glass tube of $1 \mathrm{~mm}$ in diameter (Sutter glass puller, PI1000). To measure the displacements of the carrier, a laser (Camero, $4 \mathrm{~mW}, 635 \mathrm{~nm}$ wavelength) and a quadrant photodiode detector (Silicon Sensor, QP5.8-6 SD) are placed behind the frame.

The electrical connection to the moving armature actuator is provided by a tungsten incandescent lamp filament (Fig. 4). The voltages applied to the fixed armatures, $v_{n}$ and $-v_{n}$, were provided by two high voltage operational amplifiers $( \pm 200 \mathrm{~V}$, APEX, PA82J). The reference for each amplifier are digitally set trough a DAC/ADC card (National Instrument, PCI-6259) and the control algorithm is implemented on a PC running RTAI real-time Linux kernel. The sampling rate is set to $1 \mathrm{kHz}$.

\section{CAlibration OF THE SENSOR}

The calibration of the system as a force sensor requires three parameters: the optical lever calibration constant, the stiffness of the suspension, and the electromechanical constant of the actuator. Moreover, dynamic properties of the sensor are investigated by experimental identification.

\section{A. Optical lever}

Fig. 5 shows the position of the carrier as a function of the output voltage, $U_{h}$, of the photodiode and $U_{h}$ as a function of the excitation voltage, $U$. Experimental data were fitted with straight lines. Calling $k_{1}$ and $k_{2}$ the calibration constants of the photodiode and of the actuator respectively, led to $k_{1}=387 \mu \mathrm{m} / V$ and $k_{2}=0.0274$. The relation between the $U_{h}$ and $U$ is linear as expected.

\section{B. Stiffness}

The suspension stiffness is found by measuring the natural frequency of the mass-spring system and the known mass of the carrier. For the particular tuning used in the experiments described in the Section $\mathrm{V}$, the measured natural frequency is $9.4 \mathrm{~Hz}$. Given that the natural frequency is given by $f_{0}=\frac{1}{2 \pi} \sqrt{k / m}$ and the mass is $m=1.2 \mathrm{~g}$, the stiffness, $k$, is $3.83 \mathrm{~N} / \mathrm{m}$.
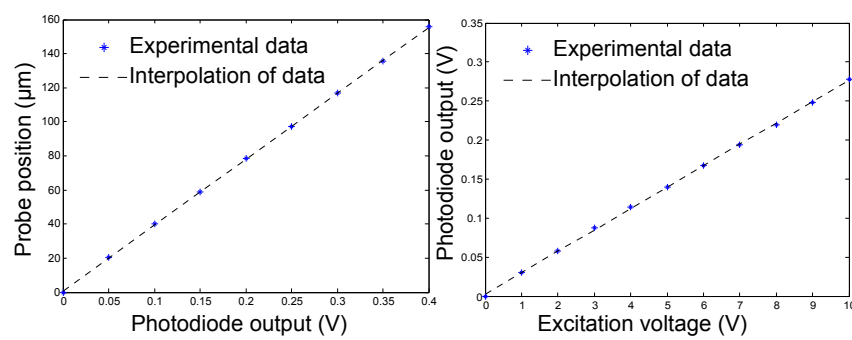

Fig. 5. Photodiode and actuator responses.

\section{Actuator}

At rest, the actuator force balances the elastic force of the suspension, thus $a_{n}=k k_{1} k_{2} U$. According to this expression the actuator constant is equal to $k k_{1} k_{2}$, here $k_{a}=40.68 \mu \mathrm{N} / \mathrm{V}$. The force produced by the actuator is indeed found to be proportional to the input voltage, validating its linear .

\section{Identification}

As shown in Section II-A, in the absence of interaction force $f$, the mechanical behavior of the system is described by

$$
m \ddot{x}-a_{n}-k x-b \dot{x}=0
$$

that is a system of order two, with a natural pulsation, $w_{n}$, and damping coefficient, $\zeta$. The Laplace transfer function is

$$
G(s)=\frac{k_{n} w_{n}^{2}}{s^{2}+2 \zeta w_{n} s+w_{n}^{2}},
$$

where $k_{n}, w_{n}$ and $\zeta$ are to be defined.

The system is identified from a step response and the values $k_{n}=0.032, \zeta=0.0047$, for $w_{n}=64 \mathrm{rad} / \mathrm{s}$, are found (step reference is fixed to 4 volts). Figure 6 compares the identified response to the experimental response of the system, indicating a very good match around $95 \%$.

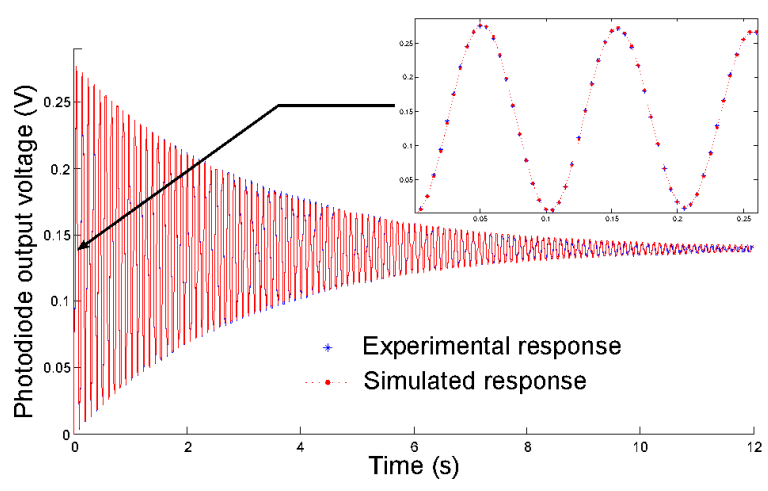

Fig. 6. Experimental and simulated step response of the prototype.

\section{ACTIVE CONTROL FOR FORCE MEASUREMENTS}

With passive sensing, the force is provided by measuring the deformation of a structural element. As the objective here is to detach the measurement from a structural stiffness, an active control approach is applied. It consists in opposing, 
by an artifice, the value of the force to be measured with an adjustable value of the same or different nature, in the objective of immobilizing the probe at its equilibrium position. In this case, the force measurement is not computed using the signal coming from the displacement sensor, but the value of the opposed artifice used to balance the force.

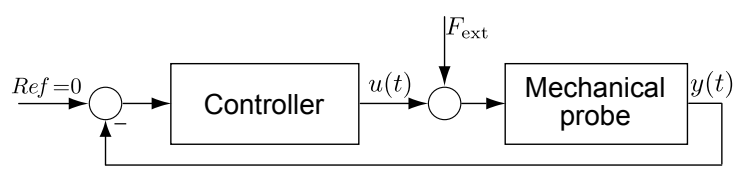

Fig. 7. Active force sensing principle.

Figure 7 illustrates the general principle of this active force measurements method. The interaction force $F_{\text {ext }}$ appears as a perturbation to be rejected by the controller in order to maintain the sensitive part of the sensor at its equilibrium position. This compensation provides directly the measure of $F_{\text {ext }}$ which is proportional to the control signal $u(t)$. This principle has many advantages :

- maintain the sensitive part of the sensor in linear range,

- improve the precision of the micro-force measurements,

- increase the force measurements range,

- improve the robustness and stability of the sensor,

\section{A. Control scheme for active force sensing}

An $H_{\infty}$ controller is used to implement this scheme. Controller output is the actuator control voltage $u(t)$ (represents the signal $v_{n}$ defined above), and its input is the feedback given by the voltage $U_{h}$ supplied by the photodiode. The output voltage $u(t)$ allows to compute the applied external force which is balanced by the controller using actuator calibration constant. The bloc diagram of this closed-loop controller is shown in Fig 7.

Fig. 8 shows the generalized plant of the $H_{\infty}$ problem. Three weighting functions are used to get adjustable parameters for the control design. Its synthesis requires to find a rational function $K(s)$ to stabilize the closed-loop, by considering $\left(\operatorname{Ref}, e_{3}\right)$ as inputs and $\left(e_{1}, r_{2}\right)$ as signals to supervise. More details are given in [19], [20], [21], [22], [23] about $H_{\infty}$ problem and method used to resolve it.

The weighing functions, $W_{1}(s)$ and $W_{2}(s)$, respectively applied to the error signal $\varepsilon$ and the control signal $u(t)$, specify the performance that the controller should achieve [18]. The weighing function $W_{1}$ specifies a tracking error of about $1 \%$ in the low frequencies, a modulus margin of 0.6 , and a bandwidth of $60 \mathrm{rad} / \mathrm{s}$. $W_{2}$ specifies the limitations on the

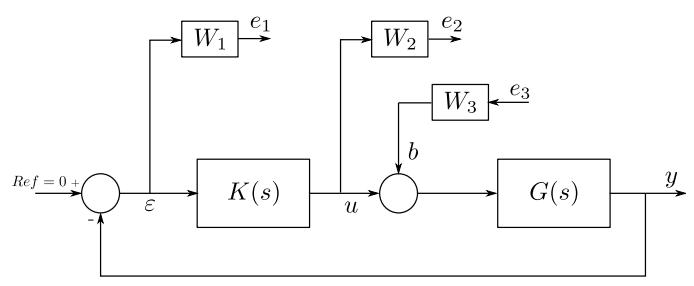

Fig. 8. Set up to determine a controller from $H_{\infty}$ optimal minimization. control signal imposed by the saturation of the amplifiers and a high cut frequency to avoid dynamics not properly modeled. The weighing function $W_{3}$ is null.

$$
W_{1}(s)=\frac{s+102}{1.7 s+0.6}, \quad W_{2}(s)=\frac{s+200}{0.001 s+20000}
$$

The $H_{\infty}$ controller, $K(s)$, is then found by minimizing the bound the $H_{\infty}$-norm from $b$ and $\operatorname{Re} f$ to $e_{1}$ and $e_{2}$. It gives the minimum value of 1.17 and a controller $K(s)$ of the fourth order. The resulting open-loop system response is shown in Fig. 9, providing interesting gain and phase margins, at $G_{m}=16.6 \mathrm{~dB}$ and $P_{m}=67.4 \mathrm{deg}$ respectively.

$$
K(s)=\frac{78.4 s^{3}+1.56810^{8} s^{2}+7.46810^{7} s+3.86110^{11}}{s^{4}+7516 s^{3}+2.57110^{6} s^{2}+3.12410^{8} s+1.09910^{8}}
$$

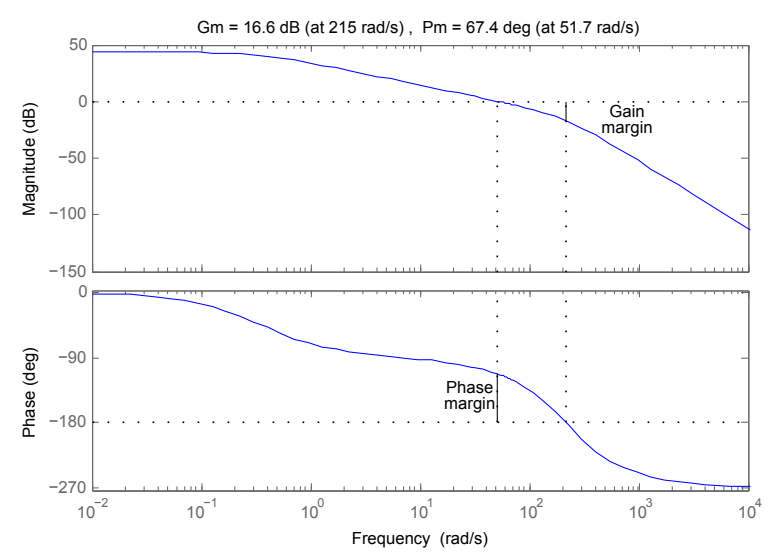

Fig. 9. Bode diagram of the open loop of the system.

The discrete time transfer function $K(z)$ using a Zero-order hold method with sampling period $h=1 \mathrm{~ms}$ is given below. This function is computable in real-time with reasonable computational effort.

$$
K(z)=\frac{2.232+11.65 z-29.95 z^{2}+16.11 z^{3}}{0.0005445-0.7045 z+2.371 z^{2}-2.667 z^{3}+z^{4}}
$$

\section{EXPERIMENTAL VALIDATION OF THE ACTIVE SENSOR}

The system is validated on a complex case for microscale force sensing, by measuring the time-course of the interaction of a thin glass probe ( $40 \mu \mathrm{m}$ in radius) with a water droplet. Such an interaction, illustrated in Fig. 10, has four main phases, namely 'approach', 'contact', 'retract', and 'contact break'.

A schematic representation of such a theoretical curve is given in Fig. 11, coming from a classical AFM probing of a hard surface. The cycle begins in $\mathrm{A}$ and terminates in $\mathrm{E}$, where the probe-surface interaction force varies as a function of the displacement of the probe, Fig. 11(a). The same behavior is represented in Fig. 11(b) where the force is shown as a function of time. This phenomenon highlights the type of interaction that are characteristic of the physics at microscopic scale. 


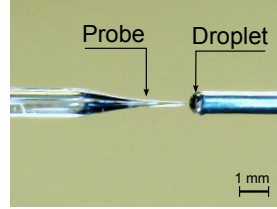

(a) approach

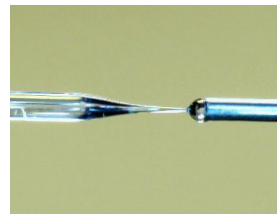

(c) retract

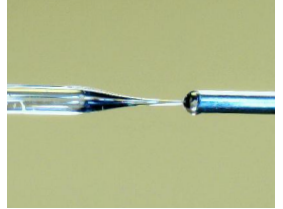

(b) contact

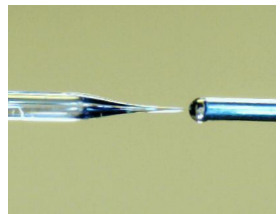

(d) contact break
Fig. 10. Interaction probe-droplet.

Two discontinuous events occur when the probe is attracted by the substrate (pull-in, point A on the plot) and when the contact is broken (pull-off, point $\mathrm{E}$ on the plot). Two types of forces are at play, attractive forces dominating in $\mathrm{B}$ and $\mathrm{D}$, and repulsive forces dominating in C [24], [25].

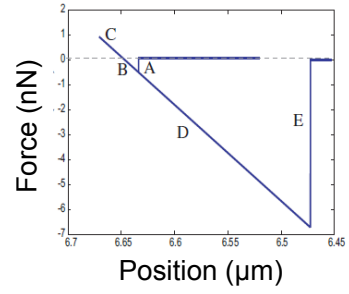

(a)

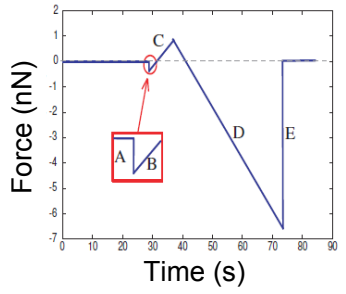

(b)
Fig. 11. Approach-retract curve. (a) force-position curve. (b) time-force curve.

Please note that this curves are theoretical representations of an interaction on a hard surface. The inherently more complex interaction on a water droplet is chosen here to depict the superior characteristics of the proposed force sensor.

As shown in Fig. 12, the experimental bench is comprised of a motorized $x y z$ micro-positioning stage for coarse motion (Sutter MP-580), an electromagnetic transducer for fine motion, a microscope (Veho-VMS-004), the laser-based optical lever, and the active probe. The bench is placed on a antivibration table.

The drop is approached to the probe, contacted and then retracted to produce an approach-retract cycle. The interaction force between the drop and the probe, during the cycle, is shown in figure 13, as a function of time. Besides, the corresponding plot of force according to the relative position of the droplet is shown in figure 14 .

As shown in figures 13 and 14, the resulting curves exemplify the characteristics of the approach-retract cycle over a droplet of water. A pull-in effort of $2 \mu \mathrm{N}$ corresponding to the instant when the drop attracts the probe is observed during the approach stage and a pull-off effort of $7 \mu \mathrm{N}$ is also observed when the contact is broken during the retract

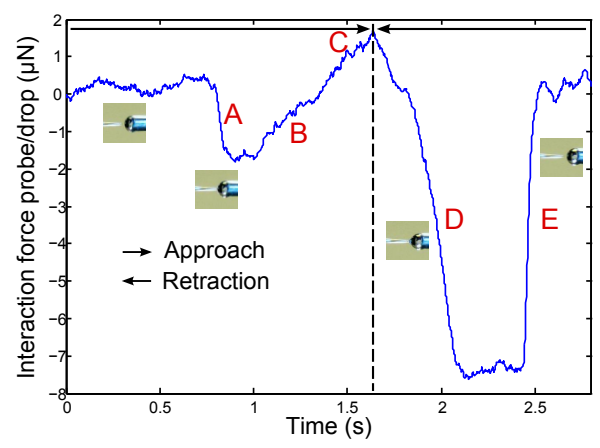

Fig. 13. Measured approach-retract curve, force vs time.

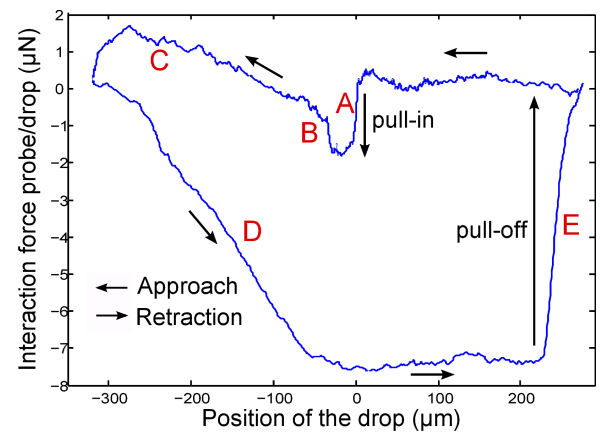

Fig. 14. Measured approach-retract curve, force vs position.

stage. Further, attractive and repulsive forces dominate the interaction. The force observed in the experiment is similar to classical approach-retract curve presented previously. However, in this case the curve is filtered by the nature of the interaction with liquid, but also, by sensor whose bandwidth is about $60 \mathrm{rad} / \mathrm{s}$ and the hysteresis ensuing from the transducer's position. This clearly represents the effectiveness and efficiency of the developed active probe system. The tests show that the resolution of the active sensing can reach $0.4 \mu \mathrm{N}$.

Nevertheless, due to the mass of the carrier $(1.2 \mathrm{~g})$ the bandwidth of the sensor is limited to $10 \mathrm{~Hz}$.

\section{Discussion AND CONCLUSIONS}

Designed around a glass fiber suspension, a differential bipolar comb actuator and an active force measurement principle, an active micro-force sensor was proposed in this paper. It is distinct from previous designs in several aspects. Actively controlled electrostatic forces are used instead of bending stresses or structural stiffness to balance the force acting on the sample. The suspension is arranged to constrain movements accurately to a single degree of freedom in translation. An electrostatic actuator combined with an optical lever for position feedback through an especially designed controller yields a large range of measurements both in force and displacement. Based on the feedback, the actuator generates an opposite balance force to cancel the action of the interaction forces. The measurement is related to the balanced force which makes the probe remains at equilibrium position. Preliminary experimental results verify 


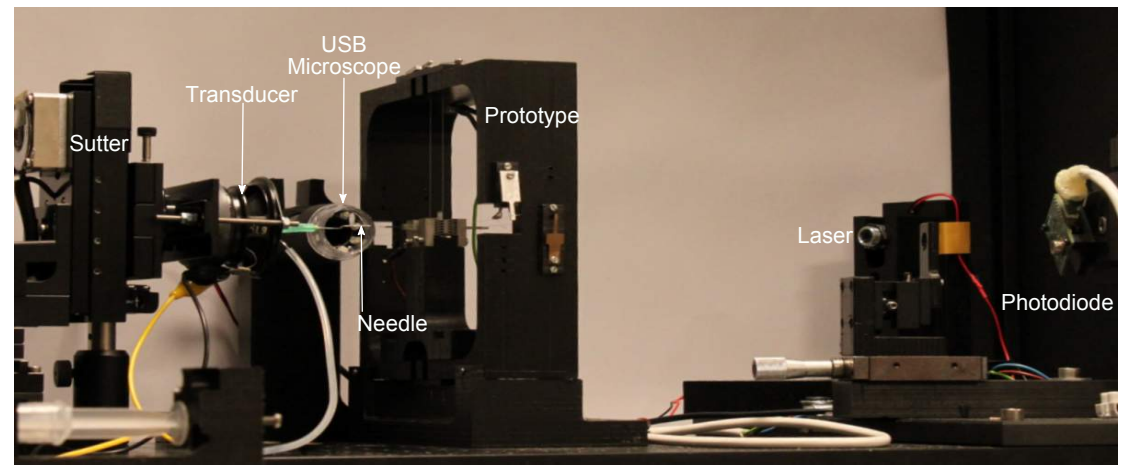

Fig. 12. Experimental bench.

the performances of the active micro-force sensor and its effectiveness.

The future work would overcome the limitations of the present prototype with improved fabrication, while retaining the same design principle. An important improvement would be the reduction of the mass of the carrier. To this end, MEMS fabrication techniques could be applied advantageously with drastic reduction in size and mass. The bandwidth of micro-scale active sensor is then expected to reach the $\mathrm{kHz}$ range.

\section{ACKNOWLEDGEMENTS}

The authors wish to thank Sheng Chao WONG for advise and help on electronic design. This work has been supported by the French National Project ROBOTEX ANR-10-EQPX44-01.

\section{REFERENCES}

[1] G. Lin, R.E. Palmer, K.S. Pister and K.P. Roos, Miniature Heart Cell Force Transducer System Implemented in MEMS Technology. IEEE Transactions on Biomedical Engineering. Vol. 48, No9, pp. 996-1006, Sept. 2001.

[2] S. Alvo, P. Lambert, M. Gauthier and S. Régnier. A van der Waals Force-Based Adhesion Model for Micromanipulation. Journal of Adhesion Science and Technology, Special Issue on Adhesion in MEMS/NEMS, Vol. 24, Nº 15-1, pp. 2415-2428, 2010.

[3] S. Haliyo, F. Dionnet and S. Régnier, Controlled rolling of micro objects for autonomous micro manipulation. International Journal of Micromechatronics, Vol. 3, N², pp. 75-101, 2006.

[4] F. Dionnet, S. Haliyo and S. Régnier, Autonomous micromanipulation using a new strategy of accurate release by rolling. Robotics and Automation, 2004. Proceedings. ICRA'04. 2004 IEEE International Conference on Robotics and Automation, Vol. 5, No 5, pp. 5019-5024, 26 April-1 May 2004.

[5] M. Sepaniak, P. Datskos, N. Lavrik and C. Tipple, Microcantilever transducers: A new approach in sensor technology. Analytical chemistry, pp. 568-575, 2002.

[6] F. Arai, A. Kawaji, T. Sugiyama, Y. Onomura, M. Ogawa, T. Fukuda, $\mathrm{H}$. Iwata and K. Itoigawa, 3d micromanipulation system under microscope. In International Symposium on Micromechatronics and Human science. pp. 127-134, 1998.

[7] Y. Sun, W. Kai-Tak, K. Roberts, J. Bischof and J. Bradley, Mechanical property characterisation of mouse zona pellucida. IEEE Transactions on nanobioscience, Vol. 2, $\mathrm{N}^{\mathrm{o}} 4$, pp. 279-285, 2003.

[8] W. Li and N. Xi, Novel micro gripping, probing and sensing devices for single-cell surgery. Engineering in Medicine and Biology Society, 2004. IEMBS '04. 26th Annual International Conference of the IEEE , Vol. 1, pp. 2591-2594, 1-5 Sept. 2004.

[9] C. M. Mate, G. M. McGuiggan, R. Erlanson and S. Chiang, Atomicscale friction of a tungsten tip on graphite surface. Phys. Lette., Vol. 59, pp. 1942-1945, Oct. 1987.
[10] H. Xie, J. Vitard, S. Haliyo, and S. Régnier, Enhanced accuracy of force application for AFM nanomanipulation using nonlinear calibration of the optical lever. IEEE Sensors Journal, Vol. 8, No 8 , pp. 14781485, 2008.

[11] P. Rougeot, S. Régnier and N. Chaillet, Forces analysis for micromanipulation. In Proceeding 2005 IEEE international symposium on computational intelligence in robotics and automation, pp. 105-110, 2005 .

[12] N. Kato, I. Suzuki, H. Kikuta and K. Iwata, Force-balancing microforce sensor with an optical-fiber interferometer. Review of scientific instruments, Vol. 68, pp. 2474-2478, 1997.

[13] K. H. Chau, S. R. Lewis, S. F. Bart, R. G. Marcheselli, and R. T. Howe, An integrated force-balanced capacitive accelerometer for lowG applications. Sens. Actuators A Phys., Vol. 54, pp. 472-276, 1996.

[14] B. P. Gogoi and C. H. Mastrangelo, A Low-voltage Force Balanced Pressure Sensor with Hermetically Sealed Servomechanism, Micro Electro Mechanical Systems, MEMS'99, pp. 493-498, 1999.

[15] Y. Sun, D. P. Potasek, D. Piyabongkarn, R. rajamani, and B. J. Nelson, Actively servoed multi-axis microforce sensors. Robotics and Automation, 2003. Proceedings. ICRA '03. IEEE International Conference on Robotics and Automation, Vol. 1, pp. 294-299, 14-19 Sept. 2003.

[16] Y. Shen, E. Winder, X. Ning, C. A. Pomeroy and U. C. Wejinya, Closed-Loop Optimal control-Enabled Piezoelectric Microforce Sensors, IEEE/ASME TRANSACTIONS ON MECHATRONICS, Vol. 11, $\mathrm{N}^{\mathrm{o}} 4$, pp. 420-427, August 2006.

[17] D. Sidobre and V. Hayward, Calibrated measurement of the behaviour of mechanical junctions from micrometre to subnanometre scale : the friction force scanner. Meas. Sci. Technol., Vol. 15, pp. 451-459, 2004.

[18] X. P. Li, and B. C. Chang, An $H_{\infty}$ design for aircraft longitudinal flight control, First IEEE Conference on Control Applications, Vol. 2, pp. 596-601, 1992.

[19] G. Cao, S. Fan and G. Xu, The Characteristics Analysis of Magnetic Bearing Based on H-infinity Controller, Proceeding of the 5th World Congress on Intelligent Control and Automation, Vol. 1, pp.752-756, June 15-19, 2004.

[20] T. Iwasaki and R. E. Skelton, All controllers for the general $h_{\infty}$ control problem : LMI existence conditions and state-space formulas. Automatica, Vol. 30, pp.1307-1317, 1994.

[21] P. Gahinet and P. Apkarian, A linear matrix inequality approach to $h_{\infty}$ control, Int. J. of Robust and non-linear control, Vol. 4, pp. 421-448, 1994.

[22] K. Glover. All optimal hankel-norm approximations of linear multivariable systems and their $l^{\infty}$ error bounds. Int. Journal of Control, Vol. 39, pp. 115-1193, 1984.

[23] J. C. Doyle, P. K. Glover, P. P. Khargonekar, and B. A. Francis. State space solutions to standard h2 and h1 control problem. IEEE Trans. Automat. Contr., Vol. 8, pp. 831-847, 1989.

[24] A. Bolopion, B. Cagneau, S. Haliyo and S. Régnier, Analysis of stability and transparency for nanoscale force feedback in bilateral coupling. Journal of Micro - Nano Mechatronics, Vol. 4, pp. 145-158, 2009.

[25] A. Bolopion, H. Xie, S. Haliyo and S. Régnier, Haptic Teleoperation for 3D Microassembly of Spherical Objects. IEEE/ASME Transaction on Mechatronics, Vol. 17, $\mathrm{N}^{\mathrm{o}} 1$, pp. 116-127, 2012. 